\title{
Natural dipeptidyl peptidase-IV inhibitor mangiferin mitigates diabetes- and metabolic syndrome-induced changes in experimental rats
}

This article was published in the following Dove Press journal:

Diabetes, Metabolic Syndrome and Obesity:Targets and Therapy

29 August 2016

Number of times this article has been viewed

\author{
Rajesh Kumar Suman' \\ Ipseeta Ray Mohanty' \\ Ujwala Maheshwari² \\ Manjusha K Borde' \\ YA Deshmukh' \\ 'Department of Pharmacology, \\ ${ }^{2}$ Department of Pathology, MGM \\ Medical College, Kamothe, Navi \\ Mumbai, India
}

\begin{abstract}
Background: Mangiferin (MNG) is known to possess antidiabetic and antioxidant activity. However, there is no experimental evidence presently available in the literature with regard to its ameliorating effects on diabetes mellitus coexisting with metabolic syndrome.

Objective: The present study was designed to evaluate the protective effects of MNG on various components of metabolic syndrome with diabetes as an essential component.

Material and methods: Adult Wistar rats were fed high-fat diets for 10 weeks and challenged with streptozotocin $(40 \mathrm{mg} / \mathrm{kg}$ ) at week three (high-fat diabetic control group). After the confirmation of metabolic syndrome in the setting of diabetes, MNG $40 \mathrm{mg} / \mathrm{kg}$ was orally fed to these rats from the fourth to tenth week.

Results: The treatment with MNG showed beneficial effects on various components of metabolic syndrome, such as reduced dyslipidemia (decreased triglyceride, total cholesterol, low-density lipoprotein cholesterol, and increased high-density lipoprotein cholesterol) and diabetes mellitus (reduced blood glucose and glycosylated hemoglobin). In addition, an increase in serum insulin, $C$-peptide, and homeostasis model assessment $\beta$ and a reduction in homeostasis model assessment of insulin resistance-IR were observed in MNG-treated group compared with highfat diabetic control group. MNG was also found to be cardioprotective (reduction in creatine phosphokinase-MB levels, atherogenic index, high-sensitivity C-reactive protein). Reduction in serum dipeptidyl peptidase--IV levels in the MNG-treated group correlated with improvement in insulin resistance and enhanced $\beta$-cell function.

Conclusion: The present study has demonstrated antidiabetic, hypolipidemic, and cardioprotective effects of MNG in the setting of diabetes with metabolic syndrome. Thus, MNG has the potential to be developed as a natural alternative to synthetic dipeptidyl peptidase-IV inhibitors beneficial in this comorbid condition.
\end{abstract}

Keywords: mangiferin, diabetes, metabolic syndrome, high-fat diet, streptozotocin, rats

\section{Introduction}

Metabolic syndrome is a constellation of closely associated disorders whose common root cause is the sedentary lifestyle, intake of calorie-rich diet, reduced physical activity, sleep deprivation, and stress. This group of symptoms comprises obesity, type 2 diabetes mellitus (T2DM), insulin resistance and hyperglycemia, hypertension, nonalcoholic fatty liver disorder, cardiovascular disorders, and dyslipidemia. ${ }^{1}$ There is a large subgroup of these metabolic syndrome patients who also have diabetic mellitus as comorbidity.

Several antidiabetic drugs are being used clinically for the management of diabetes. One of the recent additions to these antidiabetic drugs is the dipeptidyl peptidase-IV
Correspondence: Rajesh Kumar Suman Department of Pharmacology, MGM Medical College, Sector 0I, Kamothe, Navi Mumbai 410209, India

Mob +91 9699072649

Email rajesh_suman1986@hotmail.com 
(DPP-IV) inhibitors. DPP-IV inhibitors have a large number of reported effects that may be beneficial in the subgroup of metabolic syndrome with diabetes as an essential component. Although the drugs are efficacious, studies have shown that prolonged usage of these medications causes unacceptable adverse effects such as pancreatitis, angioedema, infective disorders, pancreatic cancer thyroid cancer, and severe joint pain. ${ }^{2-5}$ Moreover, these drugs are expensive as they have to be used on a regular basis for the management of chronic diseases such as diabetes. In this scenario, it would be beneficial if we could have DPP-IV inhibitors from natural sources. Various in vitro and molecular docking studies have reported about the DPP-IV inhibitory activity of medicinal extracts as well as isolated compounds.

There is a dramatic revival of interest in using natural sources in treating diabetes coexisting with metabolic syndrome due to side effects of prolonged consumption of therapeutic drugs. Asian countries such as India and the People's Republic of China are already known for their contributions toward the usage of plant medicine in preventing and overcoming problems. Mangiferin (MNG) is the major phytochemical in Mangifera indica that belongs to the family Anacardiaceae. MNG, being a glucosyl xanthone, possesses strong antioxidant, antilipid peroxidation, immunomodulation, antidiabetic cardiotonic, hypotensive, wound healing, antihyperlipidemic, antiatherogenic, and antidegenerative properties. ${ }^{6,7}$ MNG showed significant antihyperlipidemic and antiatherogenic activities as evidenced by significant alteration in lipid profile level and diminution of atherogenic index (AI) in diabetic rats. The aqueous extract of $M$. indica leaves has been reported to possess hypoglycemic activity and its active component, ${ }^{8,9} \mathrm{MNG}$, present in $M$. indica bark, was reported to produce hypoglycemic and antidiabetic activity in an animal model of genetic T2DM and in streptozotocin (STZ) diabetic rats. ${ }^{10} \mathrm{MNG}$ has been shown to counteract obesity/metabolic syndrome. It has been demonstrated that in a high-fat diet (HFD)-treated mice, mango seed kernel extract prevented weight gain and liver steatohepatitis. ${ }^{11}$ Moreover, $M$. indica extract downregulated the expression of several genes associated with the pathophysiology of obesity and inflammation, such as lipoprotein lipase, hormone-sensitive lipase, fatty acid synthase, and resistin present in liver and epididymal fat. ${ }^{12}$ Although several benefits of MNG have been reported by multiple pathways, there is no experimental evidence presently available in the literature with regard to its beneficial effect on metabolic syndrome coexisting with diabetes mellitus. In addition, the ability of MNG to modulate DPP-IV, oxidative stress, and anti-inflammatory pathway in the HFD- and low-dose STZ-induced experimental model has not been elucidated.

Hence, the present study was designed to investigate the effect of MNG on various components of metabolic syndrome with diabetes as an essential comorbidity, viz antidiabetic (blood glucose, glycosylated hemoglobin [HbA1c], serum insulin, homeostasis model assessment of insulin resistance [HOMA-IR], HOMA- $\beta$, and C-peptide), central obesity (percentage change in body weight and abdominal circumference/ thoracic circumference $[\mathrm{AC} / \mathrm{TC}]$ ratio), and hypolipidemic (lipid profile and artherogenic index) components. In addition to understand the underlying mechanisms, DPP-IV pathway (serum DPP-IV), anti-inflammatory (high-sensitivity C-reactive protein [hs-CRP]) and antioxidant (malondialdehyde [MDA]), cardioprotection (creatine phosphokinase MB [CPK$\mathrm{MB}]$ ), and beta cell preservation (insulin immunohistochemistry) properties contributing to the beneficial effects in diabetes with metabolic syndrome were also studied. Moreover, the safety parameter (pancreas [lipase, U/L]; liver [SGPT, U/L]; renal [creatinine, $\mathrm{mg} / \mathrm{dL}$ ]), function, and histopathological indices of injury were evaluated in experimental groups.

\section{Material and methods Experimental animal}

Adult male Wistar rats, 10-12 weeks old, weighing 150-200 g were used in this study. The rats were housed in the Central Animal Facility of our own MGM Medical College, Navi Mumbai, India. They were maintained under standard laboratory conditions in the animal house. The study and the study protocol were approved by MGM Medical College ethics committee, and conformed to the Committee for the Purpose of Control and Supervision of Experiments on Animals and followed the Indian National Science Academy's Guidelines for the Use and Care of Experimental Animals in Research. Rats were kept in polyacrylic cages $\left(38 \times 23 \times 15 \mathrm{~cm}^{3}\right)$ with not more than four animals per cage and housed in an air-conditioned room, kept under natural light-dark cycles. The animals were allowed free access to standard diet or HFD as the case may be and water ad libitum.

\section{Chemicals and drugs}

STZ and MNG were procured from Sigma-Aldrich Co., St Louis, MO, USA. Cholesterol was procured from Alfa Aesar, Heysham, England. All other chemicals and reagents used were of analytical grade.

\section{Preparation HFD}

The HFD was prepared indigenously in our laboratory by using normal pellet diet, raw cholesterol, mixture of vanaspati 
ghee, and coconut oil (2:1). Normal rat pellet diet was powdered by grinding and mixed with $2.5 \%$ cholesterol and mixture of vanaspati ghee and coconut oil (5\%). The mixture was made into pellet form and put into freezer to solidify. In addition, $2 \%$ raw cholesterol powder was mixed in coconut oil and administered to the rats by oral route $(3 \mathrm{~mL} / \mathrm{kg})$.

\section{Experimental model of diabetes with metabolic syndrome}

The HFD along with $2 \%$ liquid cholesterol $(3 \mathrm{~mL} / \mathrm{kg})$ was orally fed to rats for 3 weeks to induce metabolic syndrome. After 3 weeks of dietary manipulation, overnight fasted rats were injected intraperitoneally (ip) with STZ $(40 \mathrm{mg} / \mathrm{kg}$ ). The animals were allowed to drink $5 \%$ glucose solution overnight to overcome drug-induced hypoglycemia. The body weight and biochemical parameters (blood glucose, total cholesterol, and triglyceride [TG]) were estimated 7 days after the vehicle or STZ injection, ie, on 4 weeks of dietary manipulation in rats. The rats with blood glucose $(>200 \mathrm{mg} / \mathrm{dL})$, total cholesterol $(>110 \mathrm{mg} / \mathrm{dL}), \mathrm{TG}(>150 \mathrm{mg} / \mathrm{dL})$, change in body weight ( $8 \%$ of initial weight), and reduced highdensity lipoprotein (HDL) levels $(<35 \mathrm{mg} / \mathrm{dL})$ confirmed the presence of metabolic syndrome with diabetes. Thereafter, the rats were either fed normal diet or HFD as per the protocol for 10 weeks. Blood samples were collected from the retro-orbital plexus under light anesthesia at 0 week, 4 weeks, 7 weeks, and 10 weeks for estimation of biochemical parameters. At the end of experimental period, rats were sacrificed for histopathological evaluation of injury to the heart, pancreas, liver, and kidney.

\section{Experimental groups}

Group 1: normal control (NC): in NC group, rats were administered distilled water per orally using a feeding cannula for study period 10 weeks. At the end of 3 weeks, $0.01 \mathrm{M}$ citrate buffer, $\mathrm{pH} 4.5$, was injected ip to mimic the STZ injections.

Group 2: high-fat diabetic control (HF-DC): the HFD was fed to rats for 10 weeks to produce metabolic syndrome. At the end of 3 weeks, diabetes was induced by a single STZ injection $(40 \mathrm{mg} / \mathrm{kg}$ body $\mathrm{wt}$, ip dissolved in $0.01 \mathrm{M}$ citrate buffer, $\mathrm{pH} 4.5)$.

Group 3: metformin (MET): the HFD was fed to rats for 10 weeks to produce metabolic syndrome. At the end of 3 weeks, diabetes was induced by a single STZ injection (40 mg/kg body wt, ip dissolved in $0.01 \mathrm{M}$ citrate buffer, $\mathrm{pH} 4.5)$. The MET $(100 \mathrm{mg} / \mathrm{kg})$ was fed orally to rat from fifth week to tenth week daily.
Group 4: vildagliptin (VIL): the HFD was fed to rats for 10 weeks to produce metabolic syndrome. At the end of 3 weeks, diabetes was induced by a single STZ injection (40 mg/kg body wt, ip dissolved in $0.01 \mathrm{M}$ citrate buffer, $\mathrm{pH} 4.5)$. VIL (10 mg/kg) was fed orally to rat from fifth week to tenth week daily.

Group 5: MNG: the HFD was fed to rats for 10 weeks to produce metabolic syndrome. At the end of 3 weeks, diabetes was induced by a single STZ injection ( $40 \mathrm{mg} / \mathrm{kg}$ body $\mathrm{wt}$, ip dissolved in $0.01 \mathrm{M}$ citrate buffer, $\mathrm{pH} 4.5)$. The MNG (40 mg/kg) was fed orally to rat from fifth week to tenth week daily.

\section{Evaluation parameters}

1. DPP-IV in vitro assay: DPP-IV assay was performed using DPP-IV assay kit procured from Sigma-Aldrich Co., St Louis, MO, USA. In this assay, DPP-IV activity was determined by the cleavage rate of 7-amino4-methylcoumarin (AMC) from the synthetic substrate H-glycyl-prolyl-AMC. One unit of DPP-IV is the amount of enzyme that hydrolyzes the DPP-IV substrate to yield $1.0 \mathrm{U} \mathrm{mol}$ of $\mathrm{AMC} / \mathrm{min}$ at $37^{\circ} \mathrm{C}$. The standard curve for free AMC was generated using 0-50 mM AMC (SigmaAldrich Co.). DPP-IV activity was expressed as the amount of cleaved AMC per minute per $\mathrm{mL}(\mathrm{nmol} / \mathrm{min} /$ $\mathrm{mL})$. The $1 \%(\mathrm{w} / \mathrm{v}) \mathrm{MNG}$ in distilled water was used for the assay. While sitagliptin and VIL were used as a reference drugs, and control was prepared without inhibitors/test agent. Experiments were done in triplicates. A decrease in DPP-IV activity is measured for inhibition. The percentage inhibition was calculated using the following formula:

$$
\% \text { Inhibition }=\frac{\text { Control }- \text { Inhibitor }}{\text { Control }} \times 100
$$

2. Anthropometric parameter: percentage change in body weight and AC/TC ratio were recorded every 4 weeks. Percentage change in body weight was expressed according to the following equation:

$$
\begin{aligned}
& \% \text { change in body weight }=\text { Final weight }- \\
& \text { Initial weight } \times 100 / \text { Initial weight }
\end{aligned}
$$

3. Biochemical parameters: the rat blood samples of all experimental groups were collected from the retro-orbital plexus under light anesthesia at 0 week, 4 weeks, 7 weeks, and 10 weeks for estimation of blood glucose, TCh, TG, and CPK-MB. In addition, after the completion of the experimental duration (10 weeks), serum was used for the determination of the following parameters such as lipid profile, 
serum insulin, HOMA-IR, HOMA- $\beta$, C-peptide, hs-CRP, MDA, serum DPP-IV, serum glutamate pyruvate transminase (SGPT), creatinine by Auto-analyzer or ELISA kits in the pathology (NABL accredited), and pharmacology laboratory. Both insulin resistance and $\beta$-cell function were calculated by ${ }^{13} \mathrm{HOMA}-\mathrm{IR}=($ serum glucose, $\mathrm{mmol} / \mathrm{L} \times$ serum insulin, $\mu \mathrm{IU} / \mathrm{mL}) / 22.5$ and $\beta$-cell function $=($ serum insulin, $\mu \mathrm{IU} / \mathrm{mL} \times 20) /($ serum glucose, $\mathrm{mmol} / \mathrm{L})-3.5$. Lowdensity lipoprotein (LDL) is a calculated parameter, which was calculated using the Friedwald's formula, TCh - HDL/ (TG/5), and AI was calculated using the following formula $\mathrm{AI}=\mathrm{TCh}-\mathrm{HDL}-\mathrm{Ch} / \mathrm{HDL}-\mathrm{Ch}$.

4. Histopathological studies: at the end of the experiment (10 weeks), the animals were sacrificed. The heart, pancreas, liver, and kidney were immediately fixed in $10 \%$ buffered neutral formalin solution. The tissues was carefully embedded in molten paraffin with the help of metallic blocks, covered with flexible plastic molds, and kept under freezing plates to allow the paraffin to solidify. Cross sections ( $5 \mathrm{~mm}$ thick) of the fixed tissues were cut. These sections were stained with hematoxylin and eosin $(\mathrm{H} \& \mathrm{E})$ and visualized under light microscope to study the microscopic architecture of the tissues. The investigator performing the histological evaluation was blind to biochemical results and to treatment allocation (H\&E 40×).

5. Immunohistochemical localization of insulin: the pancreas was immediately fixed in $10 \%$ buffered neutral formalin solution after scarification (10 weeks). The tissues were carefully cut, $3 \mathrm{~mm}$ thick, and obtained on poly-L-lysine-coated slides and transferred to three changes of xylene for 30 minutes, followed by rehydrating with decreasing grades of alcohol. The antigen retrieval was in microwave oven, $800 \mathrm{~W}$ for 10 minutes, $420 \mathrm{~W}$ for 10 minutes, and $360 \mathrm{~W}$ for 5 minutes in citrate buffer $\mathrm{pH}$ 6. Immunostaining was done by peroxidase block with 3\% hydrogen peroxide in methanol for 5 minutes and incubated sections for 10 minutes. Primary antibody incubation was undertaken for 30 minutes at room temperature and thereafter incubated with super enhancer for 10 minutes. The tissues were incubated with poly-Horseradish peroxidase for 30 minutes followed by substrate 3,3'-diaminobenzidine. The slides were then visualized under light microscope to study the immunohistochemical localization of insulin $(40 \times)$.

\section{Statistical analysis}

The data were analyzed by one-way analysis of variance and values were considered at $P<0.05$.

\section{Results \\ DPP-IV in vitro assay of $\mathrm{MNG}$}

The MNG and synthetic DPP-IV inhibitors (sitagliptin and VIL) were screened for DPP-IV inhibitory activity in vitro assay using ELISA kit. DPP-IV inhibitory activity of synthetic drugs VIL and sitagliptin were found to be $90 \% \pm 7 \%$ and $84 \% \pm 8 \%$, respectively. The DPP-IV inhibitory activity of MNG was found to $89 \% \pm 8 \%$, which is comparable to DPP-IV inhibitory activity of reference standards VIL and sitagliptin (Figure 1). To our knowledge, the DPP-IV in vitro assay of MNG was reported for the first time in the present study.

\section{Effect of MNG on anthropometric parameters}

HFD with low dose of STZ-induced T2DM coexisting with metabolic syndrome was associated with alteration in the anthropometric and biochemical parameters (increased blood glucose, TG, and total cholesterol), which resulted in obesity, dyslipidemia, and T2DM. The percentage change in body weight in normal control (NC) was found to be 50; HF-DC, 40; MET, 20; VIL, 29, and MNG, 44. However, chronic treatment of MNG for 10 weeks significantly $(P<0.05)$ restored the body weight loss compared to the MET and VIL. However, there was no statistical difference between AC/thoracic circumference (TC) ratio of different experimental group rats (Figure 2; Table 1)

\section{Effect of MNG on biochemical parameters}

\section{Metabolic parameters}

HFD/STZ treatment resulted in significant $(P<0.001)$ elevation of blood glucose, TG, and total cholesterol levels and reduction in HDL cholesterol (HDL-Ch) levels compared

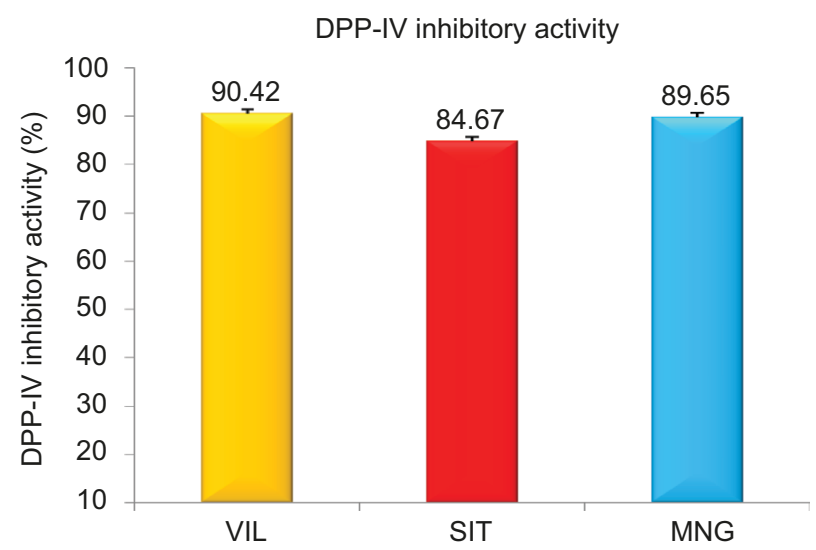

Figure I DPP-IV in vitro activity of mangiferin and standard synthetic DPP-IV inhibitor (vildagliptin and sitagliptin).

Note: Error bars are presented as SD.

Abbreviations: DPP-IV, dipeptidyl peptidase-IV; MNG, mangiferin; VIL, vildagliptin; SIT, sitagliptin; SD, standard deviation. 


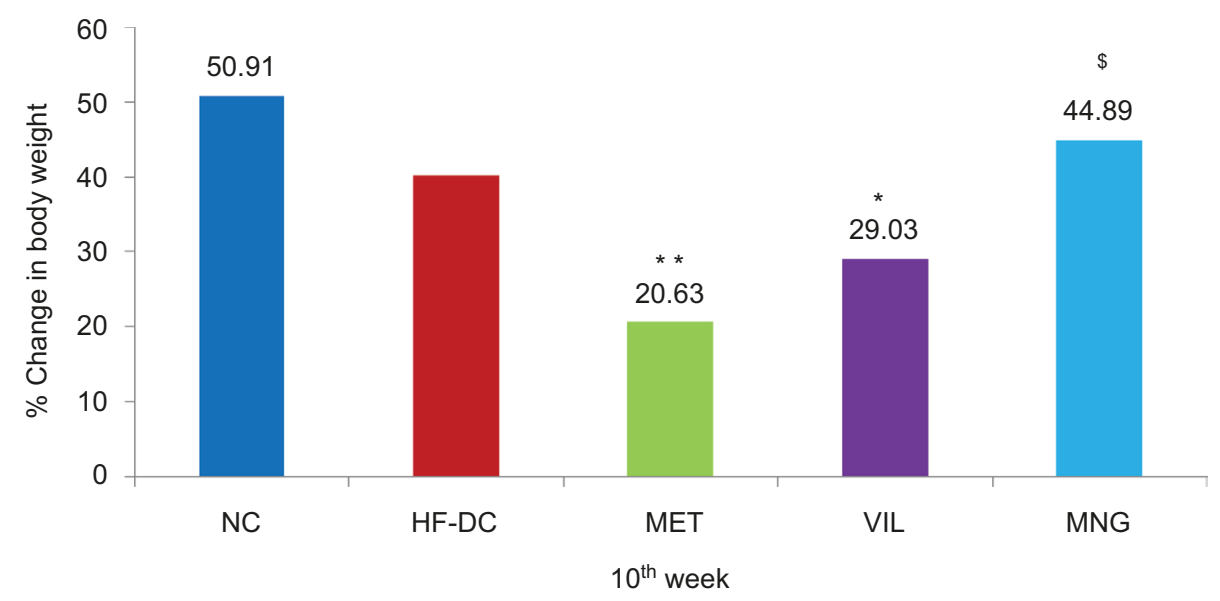

Figure 2 Percentage of change in body weight NC $(n=8)$, HF-DC $(n=7)$, MET $(n=8)$, VIL $(n=8)$, and MNG $(n=7)$.

Notes: Values are expressed as mean $\pm S D$. $* P<0.05$. $* * P<0.0$ I vs HF-DC. ${ }^{\$} P<0.05$ vs MET.

Abbreviations: NC, normal control; HF-DC, high-fat diabetic control; MET, metformin; VIL, vildagliptin; MNG, mangiferin.

Table I Anthropometric and lipid profile parameters in various experimental groups

\begin{tabular}{|c|c|c|c|c|c|c|}
\hline SN & Variable & NC & HF-DC & MET & VIL & MNG \\
\hline 1 & $\mathrm{AC} / \mathrm{TC}$ ratio & 1.06 & 1.06 & 1.06 & 1.07 & 1.06 \\
\hline 2 & $\mathrm{TCh}(\mathrm{mg} / \mathrm{dL})$ & $64 \pm 12$ & $316 \pm 34$ & $105 \pm 13 * * *$ & $98 \pm 8 * * *$ & $85 \pm 17^{* * *, \$}$ \\
\hline 3 & TG (mg/dL) & $74 \pm 17$ & $364 \pm 33$ & $|4| \pm 18 * * *$ & $130 \pm 16 * * *$ & $85 \pm 17 * * * . \$$ \\
\hline 4 & HDL (mg/dL) & $32 \pm 2$ & $25 \pm 2$ & $35 \pm 2 * *$ & $38 \pm 3 * *$ & $40 \pm 3 * * *, \$, @$ \\
\hline 5 & $\mathrm{LDL}(\mathrm{mg} / \mathrm{dL})$ & $17 \pm 1$ & $62 \pm 15$ & $34 \pm 3.16 * *$ & $32.42 \pm 2.94 * *$ & $25.08 \pm 2.28 * * . \$$ \\
\hline 6 & Atherogenic index & $0.9 \pm 0.2$ & $12 \pm 2$ & $2 \pm 0.4^{* *}$ & $1.6 \pm 0.5^{* *}$ & $1.3 \pm 0.5 * * . \$$ \\
\hline
\end{tabular}

Notes: NC $(n=8)$, HF-DC $(n=7)$, MET $(n=8)$, VIL $(n=8)$, and MNG $(n=7)$. Values are expressed as mean $\pm S D$. $* * * P<0.00 I$. $* * P<0.01$ vs HF-DC. ${ }^{\$} P<0.05$ vs $M E T$. @ $P<0.05$ vs VIL. $P$ value obtained from anova.

Abbreviations: AC/TC, abdominal circumference/thoracic circumference; NC, normal control; HF-DC, high-fat diabetic control; MET, metformin; VIL, vildagliptin; MNG, mangiferin; HDL, high-density lipoprotein; LDL, low-density lipoprotein; TG, triglyceride; TCh, total cholestrol.

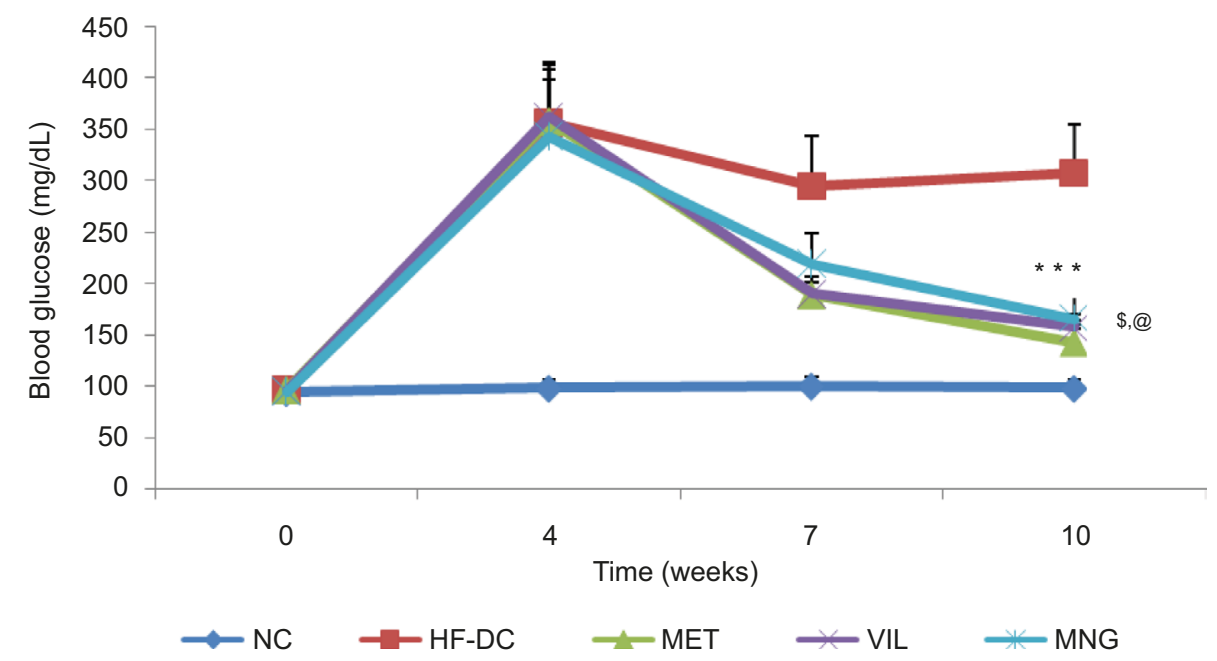

Figure 3 Time course changes in blood glucose of NC (n=8), HF-DC ( $n=7)$, MET $(n=8)$, VIL $(n=8)$, and MNG $(n=7)$.

Notes: Values are expressed as mean \pm SD. $* * * P<0.01$ vs HF-DC. ${ }^{\$} P<0.05$ vs MET. ${ }^{@ P} P<0.05$ vs VIL.

Abbreviations: NC, normal control; HF-DC, high-fat diabetic control; MET, metformin; VIL, vildagliptin; MNG, mangiferin.

to the $\mathrm{NC}$ rats as noted at different periods of the study. As shown in Figure 2, MET significantly decreased the high glucose levels followed by VIL and MNG treatment compared to HF-DC (Figure 3). Similar results were also observed in the $\mathrm{HbA1c}$ levels (Figure 4).
Serum insulin was significantly $(P<0.001)$ decrease in HF-DC group compared with $\mathrm{NC}$ group at the end of 10 weeks. HOMA-IR increased in HF-DC, whereas HOMA-b reduced significantly in HF-DC group compared with NC. MET, VIL, and MNG reduced the HOMA-IR 


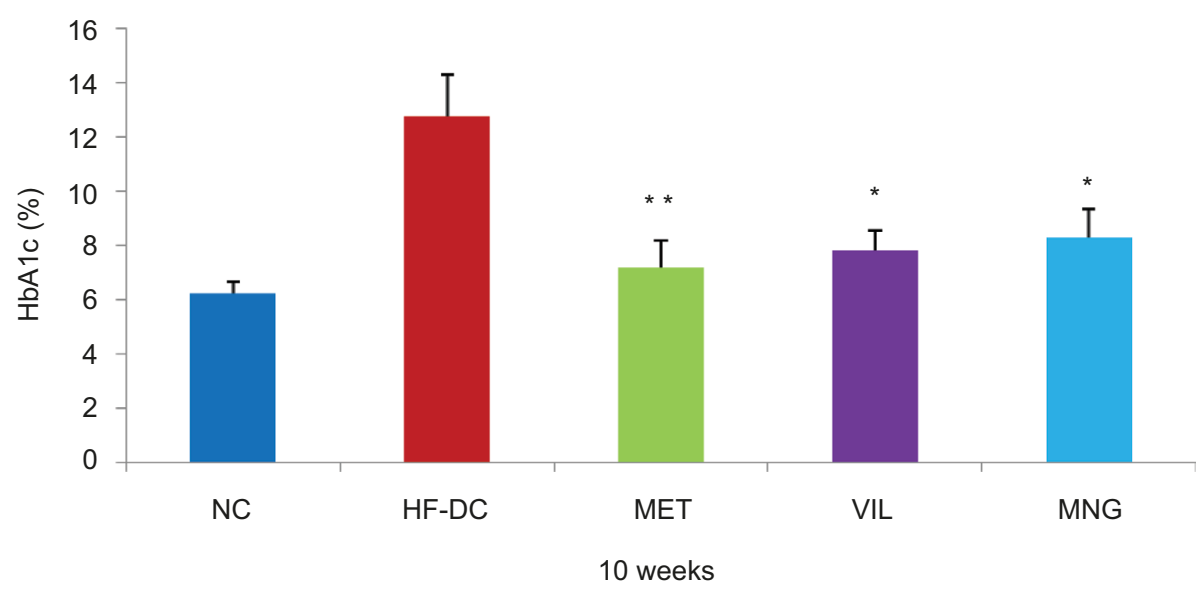

Figure 4 The HbAlc level at tenth week of NC ( $n=8)$, HF-DC ( $n=7)$, MET $(n=8)$, VIL $(n=8)$, and MNG $(n=7)$.

Notes: Values are expressed as mean \pm SD. $* * P<0.01, * P<0.05$ vs HF-DC.

Abbreviations: NC, normal control; HF-DC, high-fat diabetic control; MET, metformin; VIL, vildagliptin; MNG, mangiferin.

Table 2 Assessment of insulin, C-peptide, DPP-IV pathway, inflammatory, and oxidant variables in various experimental groups

\begin{tabular}{lllllllll}
\hline SN & $\begin{array}{l}\text { Name of } \\
\text { parameter }\end{array}$ & $\begin{array}{l}\text { Insulin } \\
(\mu \mathrm{U} / \mathrm{ml})\end{array}$ & $\begin{array}{l}\text { C-peptide } \\
(\mathbf{n g} / \mathbf{m l})\end{array}$ & HOMA-IR & HOMA- $\beta$ & $\begin{array}{l}\text { Serum DPP-IV } \\
(\lambda \mathbf{U} / \mathbf{m L})\end{array}$ & hs-CRP (mg/dL) & MDA (nmol/mL) \\
\hline $\mathrm{I}$ & NC & $6 \pm 0.6$ & $0.07 \pm 0.02$ & 1.57 & 66.6 & $4.76 \pm 0.43$ & $0.86 \pm 0.07$ & $1.84 \pm 0.08$ \\
2 & HF-DC & $2.93 \pm 1.11$ & $0.05 \pm 0.03$ & 2.17 & 5.9 & $44.53 \pm 4.5$ & $2.2 \pm 0.5$ & $6.07 \pm 0.66$ \\
3 & MET & $4.33 \pm 1.2^{* *}$ & $0.07 \pm 0.02$ & 1.64 & $17.2^{*}$ & $28.45 \pm 2.9 * *$ & $1.68 \pm 0.31^{*}$ & $4.25 \pm 0.43^{*}$ \\
4 & VIL & $4.73 \pm 0.3 I^{* *}$ & $0.06 \pm 0.03$ & 1.79 & $18.37^{*}$ & $12.32 \pm 1.02^{* * * . \$}$ & $0.94 \pm 0.16^{*}$ & $4.09 \pm 0.46^{*}$ \\
5 & MNG & $4.13 \pm 0.4^{* *}$ & $0.07 \pm 0.01$ & 1.6 & $17.08^{*}$ & $15.26 \pm 1.8^{* * * . \$}$ & $1.01 \pm 0.24^{*}$ & $3.31 \pm 0.2 I^{* *}$ \\
\hline
\end{tabular}

Notes: NC ( $n=8)$, HF-DC $(n=7)$, MET $(n=8), V I L(n=8)$, and MNG $(n=7)$. Values are expressed as mean \pm SD. *significant $P<0.05$, $* * P<0.01, * * * P<0.00 I$ vs HF-DC. ${ }^{\$} P<0.05$ vs MET. Abbreviations: DPP-IV, dipeptidyl peptidase-IV; HOMA- $\beta$, homeostasis model assessment- $\beta$; HOMA-IR, homeostasis model assessment of insulin resistance; hs-CRP, highsensitivity C-reactive protein; MDA, malondialdehyde; NC, normal control; HF-DC, high-fat diabetic control; MET, metformin; VIL, vildagliptin; MNG, mangiferin; SN, serial number; $\lambda \mathrm{U} / \mathrm{mL}$, micro unit $/ \mathrm{ml}$.

score in HFD/STZ-treated rats. In addition, the treatment groups restored beta function indicated by increase in the HOMA- $\beta$ as compared with HF-DC at 10 weeks. The C-peptide levels in HF-DC were decreased, although statistically not significant compared to $\mathrm{NC}$ and other groups (Table 2).

The total cholesterol, TG, LDL, and AI were significantly $(P<0.001)$ increased in HF-DC group compared with $\mathrm{NC}$ group at the end of 10 weeks. HDL was significantly decreased in HF-DC compared with NC. In MET, VIL, and MNG group rats, TCh and TG were significantly lower $(P<0.01)$ compared to HF-DC group rats at seventh and tenth week. Total cholesterol $P<0.001$, TG $P<0.001$, LDL $(P<0.01)$, and AI $(P<0.01)$ were significantly reduced in MNG- and standard drug-treated groups as compared with HF-DC group at the end of 10 weeks. HDL was significantly $(P<0.01)$ increased in treated group rats compared with HF-DC. MNG-treated rats favorably $(P<0.05)$ modulated lipid parameter compared to METtreated rats (Table 1$)$.

\section{Cardiac variables}

The results of the study showed a marked increase $(P<0.001)$ in plasma CK-MB isoenzyme level in HF-DC rats compared to NC group at 7 weeks and 10 weeks. MET-, VIL-, and MNG-treated groups significantly $P<0.001$ reversed the HFD/STZ induced increase in CPK-MB levels. A marked protection against cardiac damage was observed as indicated by decrease in CK-MB isoenzyme level in VIL-treated rats compared to HF-DC-administered rats (Figure 5).

\section{DPP-IV pathway, anti-inflammatory, and antioxidant variables}

The serum DPP-IV levels $(P<0.001)$ increased significantly in HF-DC group rats compared to NC group rats. MET-, VIL-, and MNG-treated rats showed significant reduction in serum DPP-IV level as compared to HF-DC rats. VIL- and MNG-treated rats showed superior reduction in serum DPPIV levels compared to MET-treated rats. Similarly, inflammatory (hs-CRP; $P<0.05$ ) and oxidative stress (MDA; $P<0.01$ ) 


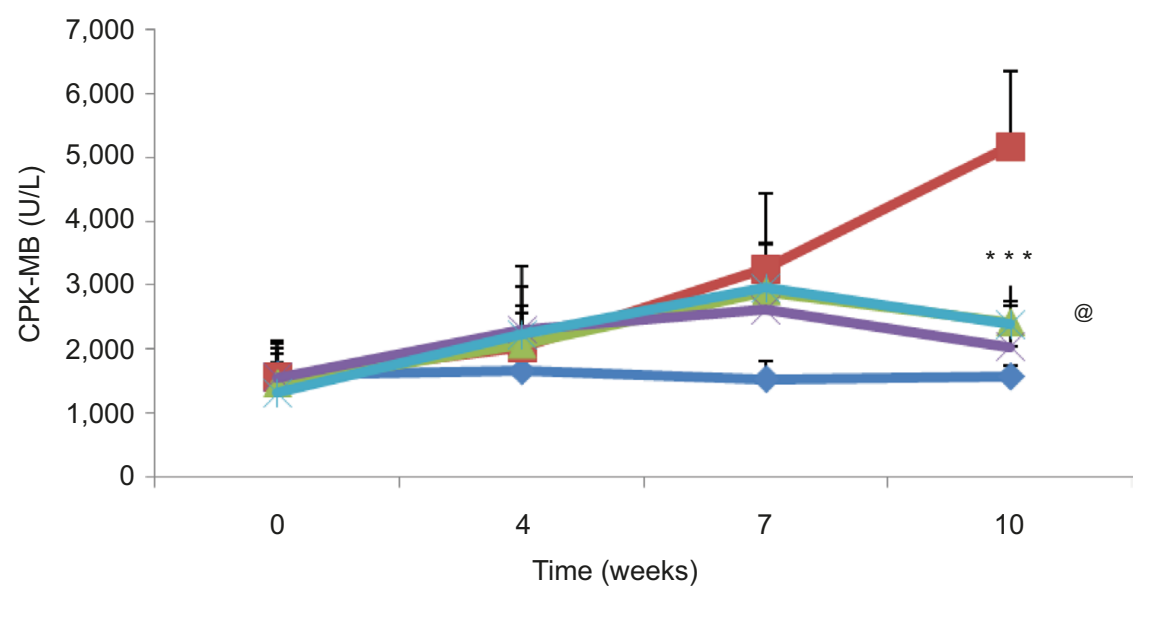

Figure 5 Time course changes in CPK-MB NC ( $n=8)$, HF-DC ( $n=7)$, MET $(n=8)$, VIL $(n=8)$, and MNG $(n=7)$.

Notes: Values are expressed as mean \pm SD. ${ }^{* * * P}<0.001$ vs HF-DC. $@ P<0.05$ vs VIL.

Abbreviations: CPK-MB, creatine phosphokinase MB; NC, normal control; HF-DC, high-fat diabetic control; MET, metformin; VIL, vildagliptin; MNG, mangiferin.

Table 3 Safety marker in various experimental groups

\begin{tabular}{lllllll}
\hline SN & Variable & NC & HF-DC & MET & VIL & MNG \\
\hline I & Pancreatic marker pancreatic & $30.66 \pm 2.10$ & $48.26 \pm 9.36$ & $36.83 \pm 1.50^{*}$ & $44.06 \pm 5.44^{* *}$ & $33.50 \pm 3.33^{*} @$ \\
& lipase (U/L) & & & & \\
2 & Liver marker SGPT (U/L) & $61.25 \pm 8.68$ & $99.85 \pm 10.38$ & $77.42 \pm 7.20^{* *}$ & $75.25 \pm 6.84^{* *}$ & $66.26 \pm 8.16^{*}, @$ \\
3 & Kidney marker creatinine $(\mathrm{mg} / \mathrm{dL})$ & $0.32 \pm 0.07$ & $1.27 \pm 0.43$ & $0.51 \pm 0.06^{*}$ & $0.58 \pm 0.04^{*}$ & $0.35 \pm 0.14^{* .9 . @}$ \\
\hline
\end{tabular}

Notes: NC ( $n=8)$, HF-DC $(n=7)$, MET $(n=8)$, VIL $(n=8)$, and MNG $(n=7)$. Values are expressed as mean \pm SD. $* P<0.05$, $* * P<0.01$ vs HF-DC. ${ }^{*} P<0.05$ vs MET. $@ P<0.05$ vs VIL. $P$ value obtained from anova.

Abbreviations: NC, normal control; HF-DC, high-fat diabetic control; MET, metformin; VIL, vildagliptin; MNG, mangiferin; HDL, high-density lipoprotein; LDL, low-density lipoprotein; SN, serial number; SGPT, serum glutamate pyruvate transminase.

markers were also significantly reduced in treatment group compared to HF-DC group rats on tenth week (Table 2).

\section{Liver, kidney, and pancreatic function markers}

As shown in Table 3, the HFD/STZ-treated groups showed markedly elevated levels of SGPT (U/L; $P<0.001)$, creatinine $(\mathrm{mg} / \mathrm{dL} ; P<0.001)$, and pancreatic lipase $(\mathrm{U} / \mathrm{L} ; P<0.001)$ at tenth week compared to NC and other treated groups. However, clinical increase in pancreatic lipase and SGPT was not significant. The treatment groups did not adversely affect the function of liver, kidney, and pancreas (Table 3).

\section{Histopathological assessment}

\section{Histopathological section of myocardium}

Photomicrograph of heart of NC group rat heart revealed the noninfracted architecture of the myocardium (Figure 6A). In contrast, HF-DC group rat heart shows fatty infiltration in myocardial cells, hemorrhage, marked edema, confluent areas of myonecrosis separation of myofibers, congested blood vessels, and inflammation compared to the NC group (Figure 6B). In the treatment group rats, occasional focal myofiber loss, inflammation, necrosis, and edema were observed. However, the degree of edema, inflammation, and necrosis was less compared to the HF-DC (Figure 6C-E; H\&E 40×).

\section{Histopathological section of pancreas}

Photomicrograph of pancreas sections of NC rats shows an organized pattern and normal architecture of islets of Langerhans and beta cells (Figure 7A). In contrast, the pancreas of HF-DC group rat shows severe degenerative changes in the pancreatic islets, damaged islets of Langerhans, reduced beta cell mass, and atrophy of beta cells, with a slight loss of nucleus and cytoplasm and more inflammatory infiltration (Figure 7B). In the treatment group rats, pancreas showed improved beta cell mass, less fibrosis, less inflammatory infiltration, and hemorrhage compared to HF-DC group (Figure 7C-E; H\&E 40×).

\section{Histopathological section of liver}

Photomicrograph of liver sections of NC rats showed normal architecture of central vein, peripheral vein, and 
A

B

C

D

E

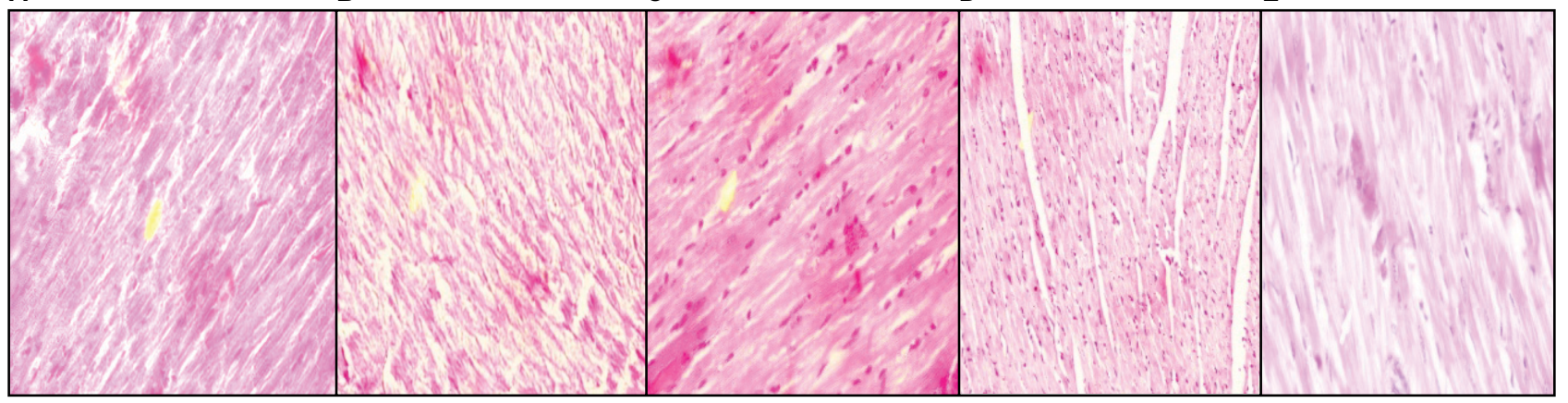

Figure 6 Histopathological section of myocardium.

Notes: (A) Photomicrograph of heart of NC group rat heart revealed the noninfracted architecture of myocardium. (B) HF-DC group rat heart shows fatty infiltration in myocardial cells, hemorrhage, marked edema, congested blood vessels, and inflammation. (C) In the MET group rats, occasional focal myofiber loss and the degree of edema, inflammation, and necrosis were less compared to the HF-DC. (D) In the VIL group rats, the degree of edema and inflammation were found but less. (E) In the MNG group rats, edema, inflammation, and necrosis were less. Magnification 40X.

Abbreviations: NC, normal control; HF-DC, high-fat diabetic control; MET, metformin; VIL, vildagliptin; MNG, mangiferin.

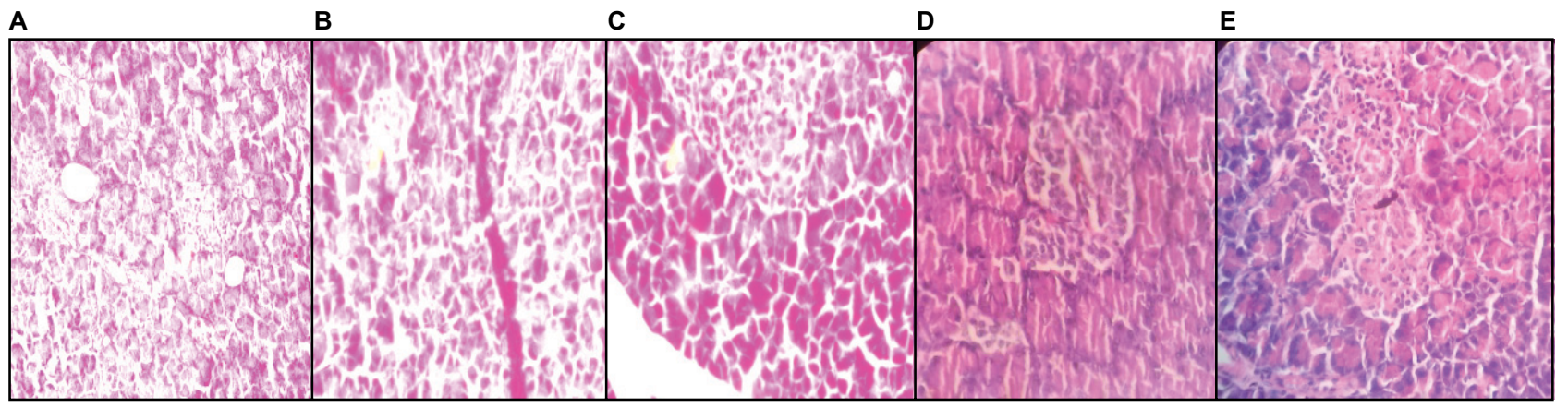

Figure 7 Histopathological section of pancreas.

Notes: (A) Photomicrograph of pancreas sections of NC rats shows an organized pattern and shows normal architecture pancreas. (B) The pancreas of HF-DC group rat shows severe degenerative changes in the pancreatic islets, damaged islets of Langerhans, reduced beta cell mass, and the atrophy of beta cells. (C) In the MET group rats, pancreas shows less inflammatory infiltration and hemorrhage compared to HF-DC group. (D) In the VIL treatment group rats, pancreas shows fibrosis, less inflammatory infiltration, and hemorrhage. (E) In the MNG group rats, pancreas shows improve beta cell mass less fibrosis, less inflammatory infiltration, and no hemorrhage. Magnification 40X.

Abbreviations: NC, normal control; HF-DC, high-fat diabetic control; MET, metformin; VIL, vildagliptin; MNG, mangiferin.

A

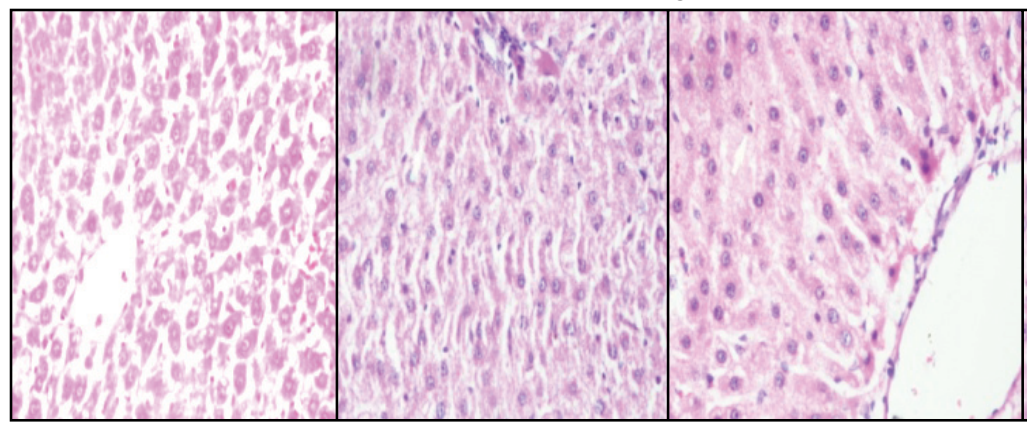

D

E

Figure 8 Histopathological section of liver.

Notes: (A) Photomicrograph of liver sections of NC rats shows normal architecture of liver. (B) In contrast, the liver of HF-DC group rat shows fatty liver, moderate fatty degeneration, ballooning of cell, inflammatory infiltration more, and congestion of blood vessels in the central vein. (C) In the MET group rats, liver shows less fatty degeneration, inflammatory infiltration, congestion of blood vessels, fibrosis, edema, and necrosis. (D) In the VIL group rats, liver shows less fatty degeneration, inflammatory infiltration, edema, and necrosis. (E) In the MNG group rats, liver shows less inflammatory infiltration, edema, and normal structure of central vein, peripheral vein, and no congestion of sinosoides. Magnification 40X.

Abbreviations: NC, normal control; HF-DC, high-fat diabetic control; MET, metformin; VIL, vildagliptin; MNG, mangiferin.

no congestion of sinosoides (Figure 8A). In contrast, the liver of HF-DC group rat showed fatty liver, moderate fatty degeneration, ballooning of cell, inflammatory infiltration, and congestion of blood vessels in central vein (Figure 8B).
In the treatment group rats, liver showed less fatty degeneration, inflammatory infiltration, congestion of blood vessels, fibrosis, edema, and necrosis compared to HF-DC group (Figure 8C-E; H\&E 40×). 


\section{Histopathological section of kidney}

Photomicrograph of kidney sections of NC rats showed normal structure of the kidney. There was absence of congestion of glomerular blood vessels, tubular necrosis, inflammation, and cloudy degeneration (Figure 9A). In contrast, histological assessment of the HF-DC group rat demonstrated congestion of glomerular blood vessels, tubular necrosis, inflammation, and cloudy degeneration compared to NC group (Figure 9B). In treatment group, kidney showed less congestion of glomerular blood vessels, less hemorrhage, less tubular necrosis, and inflammation compared to HF-DC group (Figure 9C-E; H\&E 40×).

\section{Immunohistochemistry of pancreas for insulin localization}

Immunohistochemistry of NC group pancreas showed increased localization of insulin in the NC compared to HF-DC. The HF-DC group showed loss of beta cell mass, resulting in decrease in insulin secretion. Treatment group increased the proportion of beta cell, which was functional and secreting insulin compared to HF-DC. From the quantitative immunohistochemical finding of insulin localization within parenchymal cells of pancreas, the $\mathrm{NC}$ rats showed $>85 \%$ positivity. HF-DC $<10 \%$ positivity, MET $10 \%-12 \%$ positivity, VIL $15 \%-20 \%$ positivity, and MNG $25 \%-30 \%$ positivity are noted. (Figure 10A-E, Table 4).

\section{Discussion}

The metabolic syndrome is defined by the US National Cholesterol Education Program Adult Treatment Panel III (2001) as the presence of at least three of the following criteria: central obesity: waist circumference $\geq 102 \mathrm{~cm}$ or 40 in (male) and $\geq 88 \mathrm{~cm}$ or 35 in (female); dyslipidemia: $\mathrm{TG} \geq 1.7 \mathrm{mmol} / \mathrm{L}$ $(150 \mathrm{mg} / \mathrm{dL}$ ) and HDL-Ch $<40 \mathrm{mg} / \mathrm{dL}$ (male) and $<50 \mathrm{mg} / \mathrm{dL}$ (female); blood pressure: $\geq 130 / 85 \mathrm{mmHg}$; and fasting plasma glucose: $\geq 6.1 \mathrm{mmol} / \mathrm{L}(110 \mathrm{mg} / \mathrm{dL}) .{ }^{14}$ The incidence of metabolic syndrome is rising at an unprecedented pace. A large subset of diabetic patients have coexisting metabolic

A

B

C

D

E

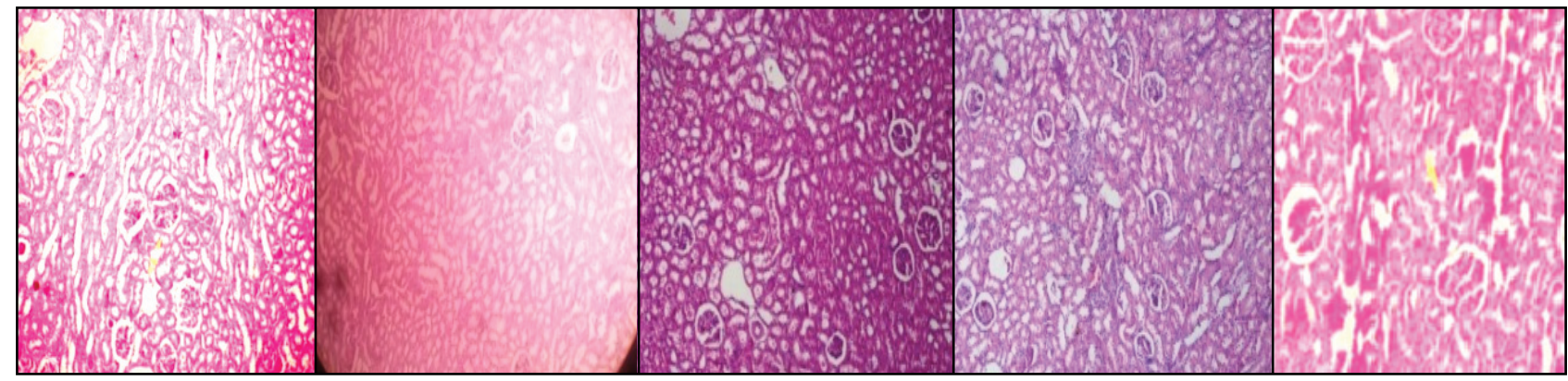

Figure 9 Histopathological section of kidney.

Notes: (A) Photomicrograph of kidney sections of NC rats shows normal structure of the kidney. (B) HF-DC group rats demonstrated congestion of glomerular blood vessels, tubular necrosis, inflammation, and cloudy degeneration. (C) In MET group, kidney shows congestion of glomerular blood vessels, less hemorrhage, less tubular necrosis, inflammation, and focal area compared to HF-DC group. (D) In VIL group, kidney shows congestion of glomerular blood vessels, inflammation, and focal area. (E) In MNG group, kidney shows no congestion of glomerular blood vessels, less tubular necrosis, and inflammation. Magnification 40X.

Abbreviations: NC, normal control; HF-DC, high-fat diabetic control; MET, metformin; VIL, vildagliptin; MNG, mangiferin.

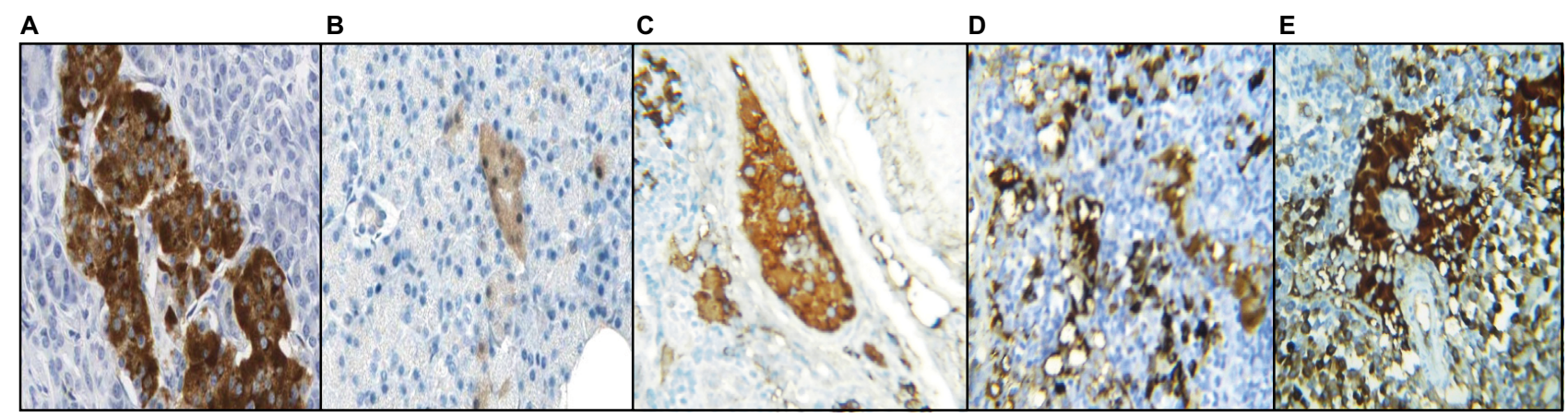

Figure 10 Immunohistochemistry of pancreas.

Notes: Immunohistochemical localization of insulin. (A) Immunohistochemistry of NC group pancreas showed increased localization of insulin. (B) The HF-DC group showed decreased localization of insulin and hence loss of beta cell functions. (C) The MET group pancreas showed increased localization of insulin compared to HF-DC group. (D) The VIL group pancreas showed increased localization of insulin compared to HF-DC group. (E) The MNG group pancreas showed marked increased localization of insulin compared to standard groups. Magnification 40X.

Abbreviations: NC, normal control; HF-DC, high-fat diabetic control; MET, metformin; VIL, vildagliptin; MNG, mangiferin. 
Table 4 Immnunohistochemistry (IHC) of pancreas for localization of insulin

\begin{tabular}{lll}
\hline SN & $\begin{array}{l}\text { Groups } \\
\text { of rats }\end{array}$ & IHC - finding for insulin \\
\hline I & NC & $>85 \%$ positivity is noted within parenchymal cells \\
2 & HF-DC & $<10 \%$ positivity is noted within parenchymal cells \\
3 & MET & $10 \%-12 \%$ positivity is noted within parenchymal cells \\
4 & VIL & $15 \%-20 \%$ positivity is noted within parenchymal cells \\
5 & MNG & $25 \%-30 \%$ positivity is noted within parenchymal cells \\
\hline
\end{tabular}

Abbreviations: NC, normal control; HF-DC, high-fat diabetic control; MET, metformin; VIL, vildagliptin; MNG, mangiferin; SN, serial number.

syndrome. Therefore, we need good experimental models that mimic the pathological features of diabetes with metabolic syndrome, which can be used to screen potential drugs beneficial in this condition. With this point of view, in the present study, a rat model that replicates features of human metabolic syndrome with T2DM was developed in the laboratory. ${ }^{15}$ This model resulted in altered anthropometric parameters, hyperglycemia, insulin resistance, hyperlipidemia, and lowgrade inflammation. Therefore, the model was selected for evaluation of the therapeutic potential of drugs in the setting of diabetes with metabolic syndrome.

DPP-IV inhibitors (gliptins) have unique benefits such as improving the secretion of insulin and glucose-dependent suppression of glucagon synthesis, which complement and extend the current available therapeutic options for T2DM. Other benefits include reduction in blood pressure and amelioration of lipid profile. As a class, the DPP-IV inhibitors in clinical practice do not cause weight gain and are weight neutral, with a very low incidence of hypoglycemia as a side effect. In addition, they have beneficial cardiovascular benefits. DPP-IV inhibitors are being used in clinical practice as second-line therapy in addition to MET in overweight and obese patients who fail to reach glycemic targets with MET monotherapy. However, some observational studies have reported increased risk of pancreatitis, pancreatic cancer associated with DPP-IV inhibitors. In addition, these drugs are expensive as they have to be used on a regular basis for the management of chronic diseases such as diabetes. In this scenario, if we could have DPP-IV inhibitors from indigenous sources that lack the undesirable effects of synthetic DPP-IV Inhibitors and are less expensive, it would be desirable.

Nature-derived phytochemicals possess several unique properties that may be useful for the management of metabolic syndrome. MNG has been shown to have antiobesity, antidiabetic, antioxidant, and anti-inflammatory properties. The present study demonstrated that MNG mitigates diabetes- and metabolic syndrome-induced changes in experimental rats by favorably modulated antidiabetic (blood glucose, $\mathrm{HbAlc}$, and restoration of pancreatic function), central obesity (body weight and $\mathrm{AC} / \mathrm{TC}$ ratio), and hypolipidemic (favorable lipid profile and artherogenic index), and cardioprotective (CPK-MB) parameters. In addition, to understand the mechanisms, DPP-IV pathway (serum DPP-IV), anti-inflammatory (CRP levels), and antioxidant (MDA) contributing to the beneficial effects of MNG in diabetes with metabolic syndrome were studied. In addition, safety parameters (pancreas [lipase, U/L], liver [SGPT, U/L], and renal [creatinine, $\mathrm{mg} / \mathrm{dL}$ ]) were assessed.

The effect of MNG on various components of metabolic syndrome is as follows.

\section{Hyperglycemia}

The present study evaluated several metabolic parameters, such as blood glucose, $\mathrm{HbAlc}$, serum insulin, and C-peptide. The blood glucose levels in the HF-DC group rats were significantly higher compared to $\mathrm{NC}$ group rats at fourth, seventh, and tenth week. Kuate et al ${ }^{16}$ also demonstrated that high-carbohydrate and -fat diet does not induce frank diabetes in experimental rats. Treatment with MNG and standard drugs MET and VIL resulted in a significant reduction in the blood glucose level in comparison to HF-DC group, respectively. Sellamuthu et a ${ }^{17}$ demonstrated that in STZ-induced diabetic mice, MNG treatment increased the activity of glycolytic and glycogen synthesis pathways, while decreased gluconeogenesis. Inhibition of gluconeogenesis in the liver reduces hepatic glucose production and thus reduces blood glucose levels. In addition to hyperglycemia, the present study results also found good glycemic control as indicated by decreased $\mathrm{HbA} 1 \mathrm{c}$ levels in MNG group compared with HF-DC. Muruganandan et al, ${ }^{6}$ reported that chronic treatment of MNG $(10 \mathrm{mg} / \mathrm{kg}$ and $20 \mathrm{mg} / \mathrm{kg}$, ip) caused significant reduction in the HbA1c levels in STZ diabetic rats further substantiating its therapeutic effects in the long-term glycemic control of diabetes mellitus. In earlier study, the potential of MNG to reduce $\mathrm{HbAl}$ c had not been studied in the setting of diabetes coexisting with metabolic syndrome model.

A deficiency of insulin and a decline in pancreatic function were evidenced by a reduced level of serum insulin, C-peptide, HOMA- $\beta$, and increased HOMA-IR respectively, in HF-DC group compared to NC group rat. In this study, treatment with MNG restored the disturbed glucose homeostasis and improved insulin sensitivity as indicated by the HOMA-IR and $\beta$-cell function, indicating that it can improve insulin resistance. Similar actions of MNG were previously reported. ${ }^{17} \mathrm{C}$-Peptide is produced in beta cells in the pancreas and secreted into the blood stream. C-peptide is considered as 
an important component in the biosynthesis of insulin and is an excellent parameter for evaluating pancreatic $\beta$-cells function. C-peptide was restored by treatment with MET, VIL, and MNG. It is speculated that MNG might possess dual beneficial effects on the pancreatic mechanism, ie, stimulating insulin release from the pancreatic cells, restoring the pancreatic beta cell mass, and reducing insulin resistance by extrapancreatic mechanisms. Such multiple actions may explain the beneficial effects of MNG. In addition, the biochemical results showed restoring effects in blood glucose with concomitant improvement in insulin and C-peptide levels in conformity with histopathological and immunohistochemical findings of MNG and standard drugs MET and VIL.

\section{Obesity}

Various anthropometric parameters, such as body weight and ratios (AC/TC), were evaluated in the NC, HF-DC, and treatment groups. Challenge with STZ caused decrease in body weight, which is typically seen in T2DM but not in metabolic syndrome. MNG treatment did not significantly alter the body weight compared to HF-DC group. In contrast, significant weight loss was observed with MET and VIL treatments.

\section{Dyslipidemia}

In our study, HFD/STZ-treated rats exhibited clear-cut abnormalities in lipid metabolism as evidenced from the significant elevation of plasma total cholesterol, TGs, LDL cholesterol, and $\mathrm{AI}$ and reduction of HDL-Ch levels in HF-DC group rats. Treatment with $\mathrm{MNG}$ and standard drugs significantly restored the elevated lipid parameter. The glucose-lowering action of the MNG can be due to the consequence of an improved lipid metabolism apart from the direct interaction with glucose homeostasis. The TGs lowering property of MNG could indirectly contribute to the overall antihyperglycemic activity through a mechanism of so-called glucosefatty acid cycle. ${ }^{18}$ The reduction in TGs following treatment with MNG could also facilitate the glucose oxidation and utilization and subsequently the reduction in hyperglycemia. A previous study by Muruganandan et $\mathrm{al}^{6}$ supported its potent antihyperlipidemic and antiatherogenic activity of MNG in diabetes rats. However, such results have not been demonstrated so far in experimental model of diabetes coexisting with metabolic syndrome.

\section{Cardiac variable}

The time course of changes in CPK-MB suggests that the deleterious cardiovascular changes are slow but progressive in nature. CPK-MB is a cardiac enzyme and its level in the serum indicates the extent of damage to the cardiac cells. These results are in keeping with our present study, since we found the close relationship between CPK-MB and heart tissue damage. Muruganandan et $\mathrm{al}^{6}$ have reported that administration of MNG significantly reduces the activity of $\mathrm{CK}$ and $\mathrm{LDH}$ in heart and also ameliorates the oxidative stress, thereby reducing cardiotoxicity. Similar to the results observed by Prabhu et $\mathrm{al}^{19}$, the present study supported the observation that $\mathrm{MNG}$ is capable of inhibiting plasma CPK-MB activity and ameliorates ISP-induced myocardial damage. ${ }^{19}$ However, cardioprotection of $\mathrm{MNG}$ has not been studied so far in the setting of diabetes with metabolic syndrome model. The cardioprotective effects of MNG as demonstrated by biochemical markers were also confirmed by histopathological assessment.

\section{Mechanism; DPP-IV pathway, inflammatory, and oxidant variables}

In the present study, HFD/STZ rats treated with MET, VIL, and MNG showed reduced serum DPP-IV levels in the setting of diabetes with metabolic syndrome. Yogisha and Raveesha ${ }^{20}$ have reported the DPP-IV inhibitory activity of methanolic extract of $M$. indica leaves in vitro. However, to our knowledge, the DPP-IV inhibitory activity of MNG, the active principle of $M$. indica, has been reported for the first time in the present study.

In addition to DPP-IV pathway, lipid peroxidationmediated tissue damage has been detected during the progress of diabetes mellitus. This is one of the specific features of chronic diabetes. In our earlier study, we found that MNG significantly reduced MDA level, a marker of lipid peroxidation in different organs, viz, heart, liver, and kidney by ameliorating changes in the antioxidant enzymes indicating its possible antioxidant activity that is advantageous in the treatment of diabetic complications. ${ }^{21} \mathrm{MNG}$ showed favorable effect on inflammatory markers hs-CRP. The anti-inflammatory activity of MNG may be attributed to its inhibitory action on potent inflammatory pathway, nuclear factor $-\kappa \beta$, and proinflammatory cytokines, interleukin- 1 and tumor necrosis factor- $\alpha .{ }^{21}$ As inflammation and ensuing oxidative stress forms a critical part of metabolic syndrome and diabetes, the observed effects may contribute to the overall beneficial effects of MNG.

\section{Safety variable}

Several studies have recently showed an impairment of pancreatic exocrine function in type 1 diabetes and T2DM. The analysis of serum/plasma pancreatic enzymes was suggested 
to provide additional informative parameters for the assessment of the chronicity and progress of the illness as well as of the response to therapy. Increased lipase levels as seen in HFD/STZ rats showed the presence of pancreatic tissue damage that was restored by MET, VIL, and MNG monotherapy. This is the first report of the effect of MNG on the pancreatic function in the experimental model of diabetes coexisting with metabolic syndrome. Metabolic syndrome is also associated with an increased risk of nonalcoholic fatty liver disease and kidney dysfunction. Chronic treatment with MNG did not adversely affect the liver and renal dysfunction in diabetic and metabolic syndrome rats, as evidenced by liver and renal function biochemical markers as well as histopathological studies. ${ }^{18}$

\section{Conclusion}

MNG mitigates diabetes with metabolic syndrome-induced deleterious effects. The DPP-IV Inhibitory, hypoglycemic, hypolipidemic, antioxidant, cardioprotective, and anti-inflammatory properties of MNG contribute to its beneficial effects.

\section{Acknowledgments}

The study is funded by the Indian Council of Medical Research vide grant no 58/2/2014-BMS. The abstract of this article has been presented in the Eighth International Conference on Recent Advances in Cardiovascular Sciences, Winnipeg, Canada, held on February 5-7, 2016, at Anand Pharmacy College, Gujarat, India.

\section{Disclosure}

The authors report no conflicts of interest in this work.

\section{References}

1. Huang PL. A comprehensive definition for metabolic syndrome. Dis Model Mech. 2009;2:231-237.

2. Matteucci E, Giampietro O. Dipeptidyl peptidase-4 inhibition: linking chemical properties to clinical safety. Curr Med Chem. 2011;18(31): 4753-4760.

3. Nicolau Ramis J, Masmiquel Comas L. The new antidiabetic agents in firing line. Safety reason or witch hunt. Endocrinol Nutr. 2012;59(1):1-8.

4. Monami M, Dicembrini I, Mannucci E. Dipeptidyl peptidase-4 inhibitors and pancreatitis risk: a meta-analysis of randomized clinical trials. Diabetes Obes Metab. 2014;16:48-56.
5. Elashoff M, Matveyenko AV, Gier B, Elashoff R, Butler PC. Pancreatitis and thyroid cancer with glucagon like peptide-1-based therapies. Gasteroenterology. 2011;141:150-156.

6. Muruganandan S, Srinivasan K, Gupta S, Gupta PK, Lal J. Effect of mangiferin on hyperglycemia and atherogenicity in streptozotocin diabetic rats. J Ethnopharmacol. 2005;97:497-501.

7. Andreu GP, Delgado R, Velho JA, Curti C, Vercesi AE. Iron complexing activity of mangiferin, a naturally occurring glucosylxanthone, inhibits mitochondrial lipid peroxidation induced by $\mathrm{Fe}(2)$-citrate. Eur J Pharmacol. 2005;513:47-53.

8. Aderibigbe AO, Emudianughe TS, Lawal BA. Evaluation of antidiabetic action of Mangifera indica in mice. Phytother Res. 2001;15:456-458.

9. Aderibigbe AO, Emudianughe TS, Lawal BA. Antihyperglycemic effect of Mangifera indica in rats. Phytother Res. 1999;13:504-507.

10. Saleh S, El-Maraghy N, Reda E, Barakat W. Modulation of diabetes and dyslipidemia in diabetic insulin-resistant rats by mangiferin: role of adiponectin and TNF- $\alpha$. An Acad Bras Cienc. 2014;86(4): 1935-1948.

11. Kobayashi M, Matsui-Yuasa I, Fukuda-Shimizu M, et al. Effect of mango seed kernel extract on the adipogenesis in 3T3-L1 adipocytes and in rats fed a high fat diet. Health. 2013;5:9-15.

12. Moreno DA, Ripoll C, Ilic N, Poulev A, Aubin C, Raskin I. Inhibition of lipid metabolic enzymes using Mangifera indica extracts. $J$ Food Agric Environ. 2006;4:21-26.

13. Matthews DR, Hosker JP, Rudenski AS, Naylor BA, Treacher DF, Turner RC. Home-ostasis model assessment: insulin resistance and $\beta$-cell function from fasting glucose and insulin concentrations in man. Diabetologia. 1985;28:412-419.

14. Expert Panel on Detection, Evaluation and Treatment of High Blood Cholesterol in Adults. Executive Summary of the Third Report of the Natural Cholesterol Education Program (NCEP) Expert panel on detection, evaluation and treatment of high blood cholesterol in adults (adult treatment panel III). JAMA. 2001;285:2486-2497.

15. Suman RK, Mohanty IR, Borde MK, Maheshwari U, Deshmukh YA. Development of an experimental model of diabetes co-existing with metabolic syndrome in rats. Adv Pharmacol Sci. 2016;2016:9463476.

16. Kuate D, Pascale A, Kengne N, Biapa CPN, Azantsa BGK, Muda WABW. Tetrapleura tetraptera spice attenuates high-carbohydrate, high-fat diet-induced obese and type 2 diabetic rats with metabolic syndrome features. Lipids Health Dis. 2015;14:50.

17. Sellamuthu PS, Muniappan BP, Perumal SM, Kandasamy M. Antihyperglycemic effect of mangiferin in Streptozotocin induced diabetic rats. J Health Sci. 2009;55:206-214.

18. Randle PJ, Garland PB, Hales CN, Newsholme EA. The glucose-fatty acid cycle, its role in insulin sensitivity and metabolic disturbances in diabetes mellitus. Lancet. 1963;1:785-789.

19. Prabhu S, Jainu M, Sabitha KE, Devi CS. Role of mangiferin on biochemical alterations and antioxidant status in isoproterenol-induced myocardial infarction in rats. J Ethnopharmacol. 2006;107:126-133.

20. Yogisha S, Raveesha KA. Dipeptidyl peptidase IV inhibitory activity of Mangifera indica. J Nat Prod. 2010;3:76-79.

21. Leiro J, Arranz JA, Yáñez M, Ubeira FM, Sanmartín ML, Orallo F. Expression profiles of genes involved in the mouse nuclear factorkappa B signal transduction pathway are modulated by mangiferin. Int Immunopharmacol. 2004;4:763-778.
Diabetes, Metabolic Syndrome and Obesity: Targets and Therapy is an international, peer-reviewed open-access journal committed to the rapid publication of the latest laboratory and clinical findings in the fields of diabetes, metabolic syndrome and obesity research. Original research, review, case reports, hypothesis formation, expert opinion and commentaries are all considered for publication. The manuscript management system is completely online and includes a very quick and fair peer-review system, which is all easy to use. Visit $\mathrm{http}: / /$ www.dovepress.com/testimonials.php to read real quotes from published authors. 